\title{
Origin of profunda femoris artery and its circumflex femoral branches: anatomical variations and clinical significance
}

\author{
Ashraf Y. Nasr ${ }^{1,2}$, Mohammed H. Badawoud², Abdulmonem A. Al-Hayani², \\ Adel M. Hussein ${ }^{2}$ \\ ${ }^{1}$ Department of Anatomy, Faculty of Medicine, Zagazig University, Zagazig, Egypt \\ ${ }^{2}$ Department of Anatomy, Faculty of Medicine, King Abdul Aziz University, Jeddah, Kingdom of Saudi Arabia \\ [Received 18 August 2013; Accepted 7 October 2013]
}

Background: Knowledge of the anatomical variations of the profunda femoris artery and its circumflex branches is important during angiographic diagnostic procedures as well as during performing surgery in the femoral region. The aim of this study was to examine the original sites, distances and variations of the profunda femoris artery and its circumflex branches.

Materials and methods: The study was conducted in the Department of Anatomy, Faculty of Medicine, King Abdulaziz University from October, 2011 to May, 2013 after the approval of the medical ethical committee. Dissections of 90 femoral triangles of 25 male and 20 female adult human cadavers were performed to demonstrate the origin and distribution of the profunda femoris artery and its circumflex branches.

Results: The profunda femoris artery mostly originated from the posterolateral aspect of the femoral artery in $42 \%$ of male limbs and in $42.5 \%$ of female limbs, from the posterior side in $24 \%$ of male and $27.5 \%$ of female limbs, from the lateral side in $20 \%$ of males and female limbs and from the posteromedial aspect in $14 \%$ of male limbs and in $7.5 \%$ of female limbs. The mean distance of origin of profunda femoris artery from the midpoint of inguinal ligament was $51.5 \pm 1.9 \mathrm{~mm}$ in right male, $49.7 \pm 1.9 \mathrm{~mm}$ in left male, $48.5 \pm 2.2 \mathrm{~mm}$ in right female and $48.9 \pm 2.2 \mathrm{~mm}$ in left female limbs. The medial and lateral circumflex arteries originated mostly from the profunda femoris artery (60\% in males; $57.7 \%$ in females) at a mean distance of $18.6 \pm 2.1 \mathrm{~mm}$ and $20.2 \pm 2.2 \mathrm{~mm}$ in right male, $19.6 \pm 1.9 \mathrm{~mm}$ and $22.5 \pm 2.3 \mathrm{~mm}$ in left male, $18.8 \pm 2.7 \mathrm{~mm}$ and $21 \pm 2.6 \mathrm{~mm}$ in right female and $19.1 \pm 2.1 \mathrm{~mm}$ and $21.7 \pm 2.6 \mathrm{~mm}$ in left female limbs, respectively. The original incidence of the medial and lateral circumflex femoral arteries from the femoral artery including the common trunk was $40 \%$ in male and $42.3 \%$ in female limbs. Conclusions: Awareness of the original sites and distances of the profunda femoris artery and its circumflex femoral branches will allow the surgeon to define the vascular pattern before performing any invasive procedure and to avoid unexpected iatrogenic injuries. (Folia Morphol 2014; 73, 1: 58-67)

Key words: profunda femoris, circumflex femoral arteries, origin and variations

Address for correspondence: Ashraf Y. Nasr, MD, Anatomy Department, King Abdulaziz University, PO Box 80205, Jeddah 21589, Kingdom of Saudi Arabia, tel: +966 (2) 6401000 ext. 20477, fax: +966 (2) 4601000 ext. 20121, e-mail: ashrafnaeem2013@gmail.com

This work is done in: Anatomy Department, Faculty of Medicine, King Abdul-Aziz University, Jeddah, Kingdom of Saudi Arabia. 


\section{INTRODUCTION}

The knowledge of the anatomical variations of the profunda femoris artery (PFA) and its medial and lateral circumflex branches is important in performing any diagnostic or/and surgical interventions in the femoral region [8]. The PFA is the largest branch of femoral artery (FA) and it is the main arterial supply of the muscles of the thigh [21].

Certain degree of variations, regarding the origin and course of PFA and its circumflex branches, has been reported in the literature $[2,6,8,9,11,15-17$, $19,22-25]$. The authors added that the knowledge of the original variations of PFA and its circumflex branches is of great significance in preventing the clinical complications that may occur in plastic and reconstructive surgery $[8,16,17,25]$.

The present study aimed to investigate the different origin patterns of the PFA and its medial and lateral circumflex branches. The distance between the origin of PFA and the midpoint of inguinal ligament; as well as the distance between the beginning of PFA and the origin of its circumflex branches were estimated.

\section{MATERIALS AND METHODS}

Ninety lower extremities of 45 adult human cadavers (25 male and 20 female) were used in the present study. Their age ranged from 30 to 70 years. The cadavers were obtained from the Department of Anatomy, Faculty of Medicine, King Abdulaziz University, Jeddah, Kingdom of Saudi Arabia. The femoral triangles of the lower limbs of the cadavers were dissected. The dissection started by incision and reflection of the skin to clean the underlying superficial fascia. Fine dissection of the superficial inguinal lymph nodes was done. The fascia lata was incised and the femoral triangles were exposed. The femoral sheath and its contents including FA were identified. The anterior wall of the sheath was excised to expose the FA and its branches. The PFA and its medial and lateral circumflex branches were dissected and identified.

The patterns of the PFA and its circumflex femoral branches were estimated, based on the site of their origin. The distance from the midpoint of inguinal ligament to the origin of PFA was measured. Also the distances from the origins of both circumflex femoral arteries and the PFA were measured with a Vernier's calliper, accuracy $0.01 \mathrm{~mm}$ (Fig. 3).

\section{Statistical analysis}

The collected data were tabulated in excel software sheets and statistically analysed using SPPS version 16.0 for window. The results were expressed as numbers and percentages regarding right and left sides in each sex. The mean \pm standard error of mean (SEM) were obtained for each measurement. Sex and side comparisons were done by using the unpaired student t-test. Chi-square was generated for the original sites of PFA as well as the original patterns of its medial and lateral circumflex femoral branches. P value $<0.05$ was considered statistically significant. This descriptive anatomical study was conducted at the Department of Anatomy, Faculty of Medicine, King Abdulaziz University from October 2011 to May 2013 after the approval of the medical ethical committee.

\section{RESULTS}

Four different original sites of the PFA were observed in the present study (Table 1). PFA mostly originated from the posterolateral aspect of FA (Fig. 1). This pattern was observed in $42 \%$ of male and in $42.5 \%$ of female limbs, while the posterior side of FA gave origin to PFA (Fig. 2) in $24 \%$ of male and in $27.5 \%$ of female limbs. Meanwhile, the PFA originated from the lateral side of FA (Fig. 3 ) in $20 \%$ of male and female limbs. PFA arose from the posteromedial side of FA (Fig. 4) in 14\% of male and in $10 \%$ of female limbs. Statistically, no significant differences were reported between the pattern of PFA origin in male, female, right and left limbs ( $p>0.05)$.

The mean distance between the origin of PFA and the midpoint of inguinal ligament was measured and listed (Table 2). This mean distance was $51.1 \pm 1.9 \mathrm{~mm}$ in the right male limbs and $49.7 \pm 1.9 \mathrm{~mm}$ in the left male limbs. While in the female cadavers, the PFA originated at a mean distance of $48.5 \pm 2.2 \mathrm{~mm}$ on the right side and $48.9 \pm 2.2 \mathrm{~mm}$ on the left side. In the right male limbs, the origins of the medial and lateral circumflexfemoral brancheswerenoticed at $18.6 \pm 2.1 \mathrm{~mm}$ and $20.2 \pm 2.2 \mathrm{~mm}$ distances, respectively from the origin of PFA. While the origins the medial and lateral circumflex arteries on the left male limbs were seen at $19.6 \pm 1.9 \mathrm{~mm}$ and $22.5 \pm 2.3 \mathrm{~mm}$ distances. In the female cadavers, the mean distance of the origins of the medial and lateral circumflex femoral arteries were observed at $18.8 \pm 2.7 \mathrm{~mm}$ and $21 \pm 2.6 \mathrm{~mm}$ distances, respectively from the origin of PFA on the right limbs, while on the left limbs, the origins of medial and lateral circumflex femoral arteries were seen at $19.1 \pm 2.1 \mathrm{~mm}$ and $21.7 \pm 2.6 \mathrm{~mm}$ distances, respectively.

Statistically, no side or sex significant differences were reported regarding the measurements of the PFA and its 2 circumflex femoral branches, where the range of 
Table 1. Sites of origin of the profunda femoris artery (PFA) from the femoral artery

\begin{tabular}{|c|c|c|c|c|c|c|}
\hline \multirow[t]{2}{*}{ Site of PFA origin } & \multicolumn{3}{|c|}{ Male limbs } & \multicolumn{3}{|c|}{ Female limbs } \\
\hline & Right (25) & Left (25) & Both (50) & Right (20) & Left (20) & Both (40) \\
\hline Posterolateral & $11(44 \%)$ & $10(40 \%)$ & $21(42 \%)$ & $9(45 \%)$ & $8(40 \%)$ & $17(42.5 \%)$ \\
\hline Posterior & $6(24 \%)$ & $6(24 \%)$ & $12(24 \%)$ & $5(25 \%)$ & $6(30 \%)$ & $11(27.5 \%)$ \\
\hline Lateral & $5(20 \%)$ & $5(20 \%)$ & $10(20 \%)$ & $5(25 \%)$ & $3(15 \%)$ & $8(20 \%)$ \\
\hline Posteromedial & $3(12 \%)$ & $4(16 \%)$ & $7(14 \%)$ & $1(5 \%)$ & $3(15 \%)$ & $4(10 \%)$ \\
\hline
\end{tabular}

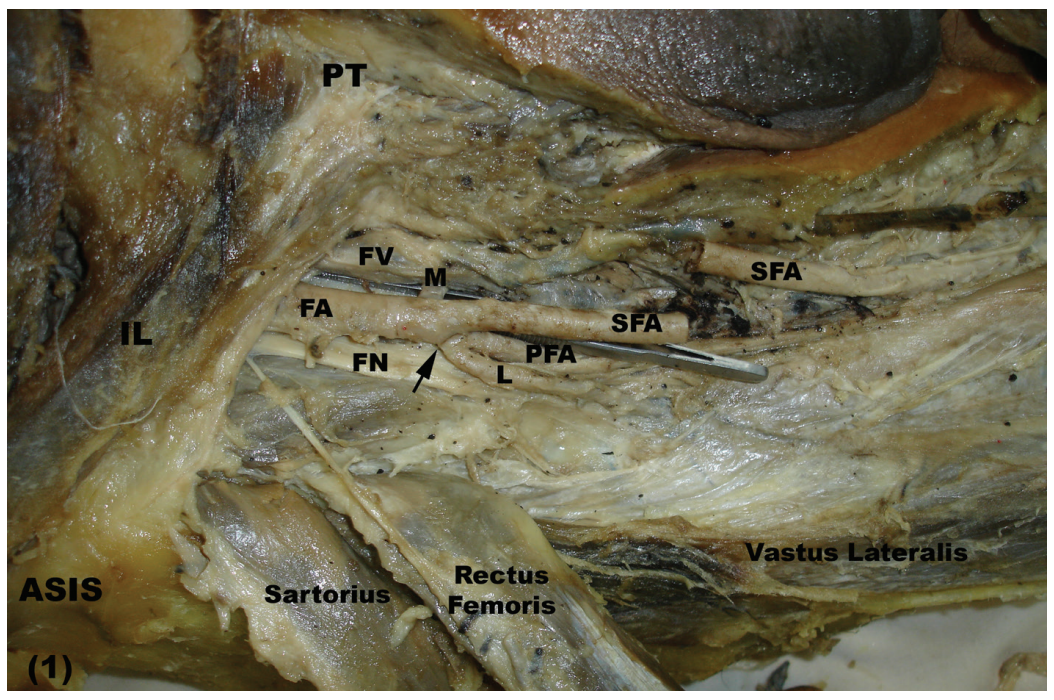

Figure 1. A right female lower limb showing the profunda femoral artery (PFA) originating from the posterolateral aspect of the femoral artery (FA) in a common stem (arrow) with the lateral circumflex femoral artery (L). The medial circumflex femoral artery (M) originates from the medial side of FA proximal to the origin of PFA. The inguinal ligament (IL) connects between the pubic tubercle (PT) and the anterior superior iliac spine (ASIS). The femoral nerve (FN) lies lateral to FA, while femoral vein (FV) lies medial to it; SFA — superficial femoral artery.

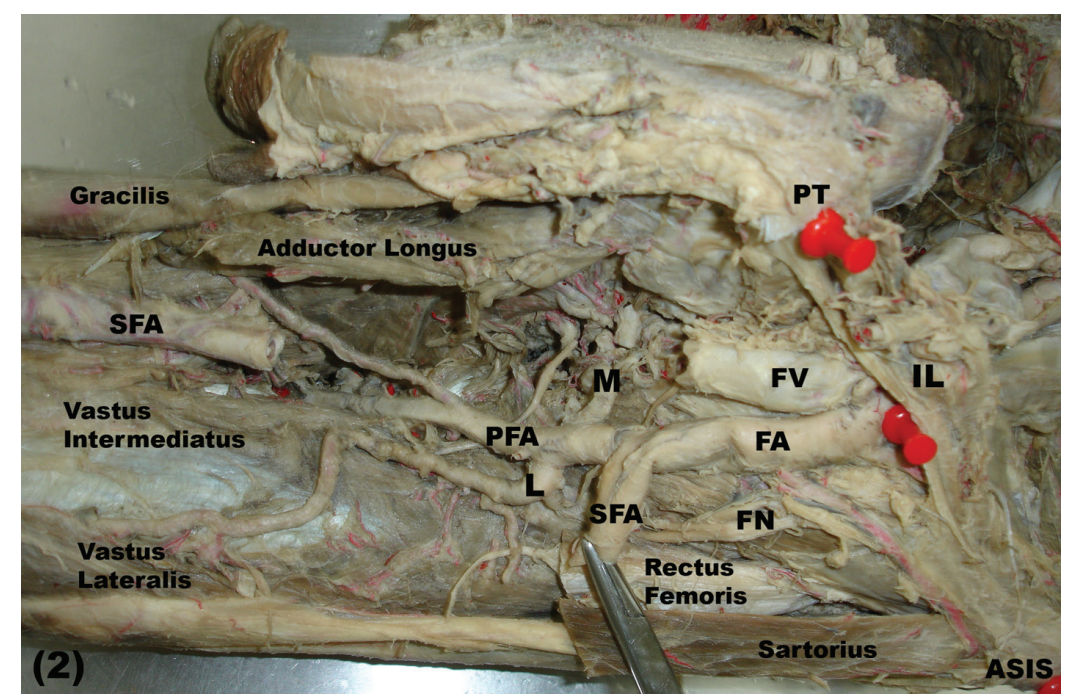

Figure 2. A left male lower limb showing the profunda femoris artery (PFA) originating from the posterior aspect of the femoral artery (FA). The medial circumflex (M) and lateral circumflex (L) femoral branches originate from their corresponding side of the PFA at the same level; PT — pubic tubercle, ASIS — anterior superior iliac spine; SFA — superficial femoral artery; FV — femoral vein; FN — femoral nerve. 


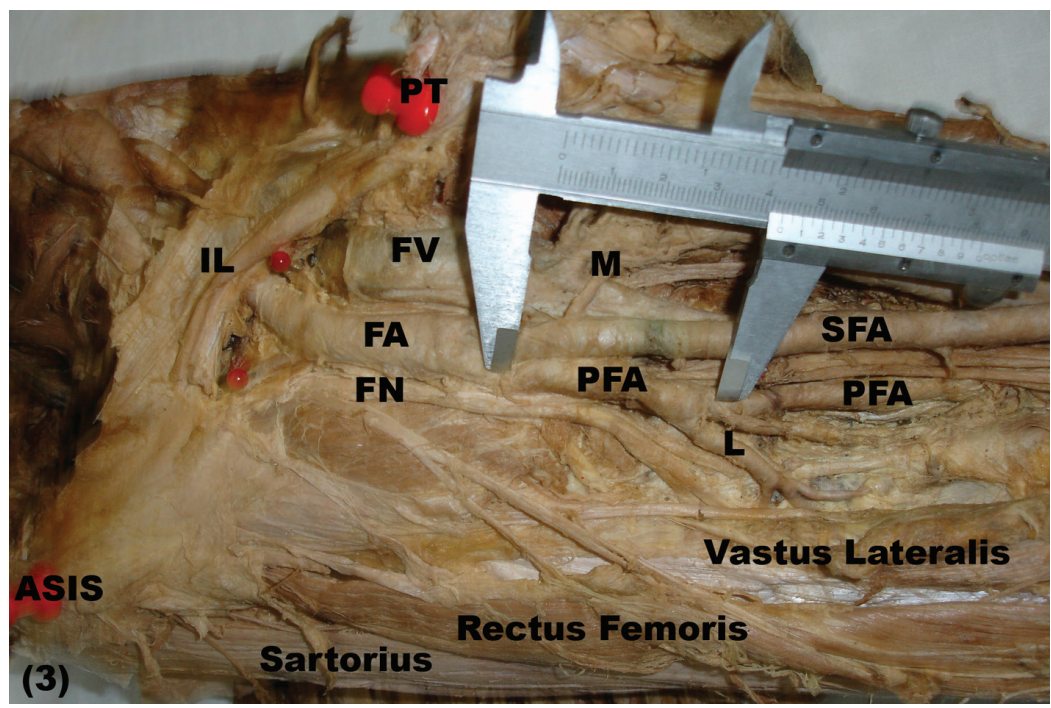

Figure 3. A right male lower limb showing the profunda femoris artery (PFA) originating from the lateral side of the femoral artery (FA). The medial circumflex femoral artery (M) originates from the superficial femoral artery (SFA) and passes medially superficial to the femoral vein (FV) while the lateral circumflex femoral artery (L) arises from the lateral side of PFA; FN — femoral nerve; IL — inguinal ligament; ASIS — anterior superior iliac spine.

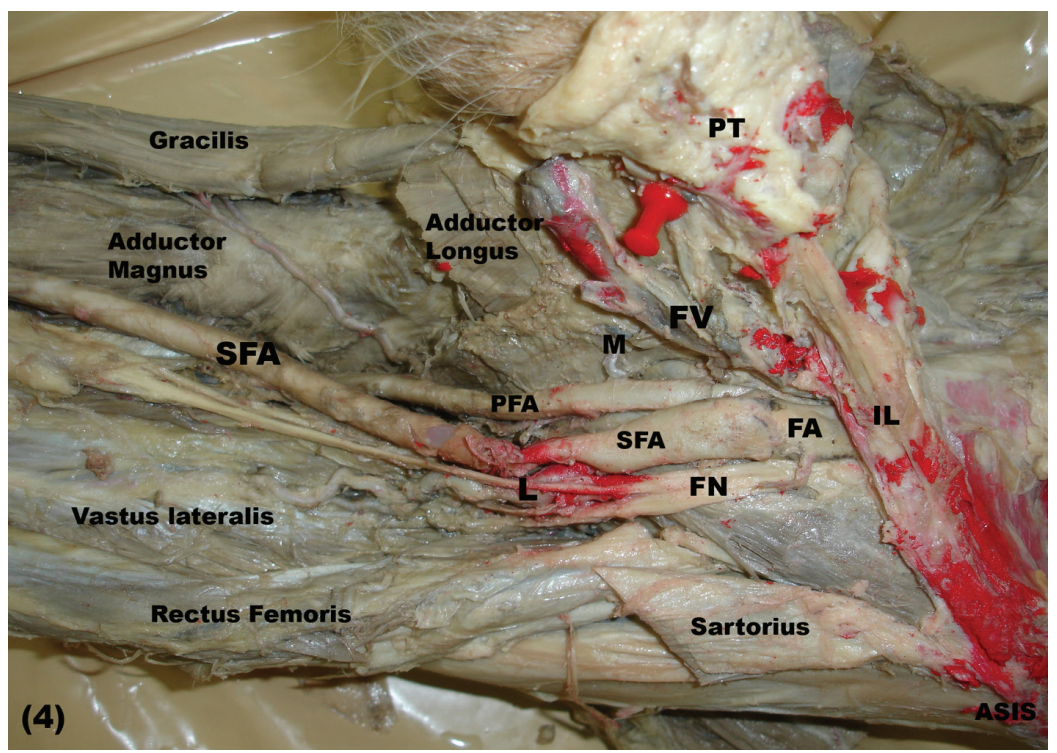

Figure 4. A left female lower limb showing a high division of femoral artery (FA) into the profunda femoris (PFA) and superficial femoral artery (SFA) branches. The PFA originates from the posteromedial aspect of the FA. The medial circumflex femoral branch (M) originates from the medial side of the profunda femoris artery, while the lateral circumflex femoral branch $(\mathrm{L})$ originates from the lateral side of the SFA; IL — the inguinal ligament; PT — pubic tubercle; ASIS — anterior superior iliac spine; FV — femoral vein; FN — femoral nerve; red colour is due to leakage of the latex.

p value was $0.31-1.00$ and that of $t$ value was $0.00-1.04$ by using the unpaired student $\mathrm{t}$-test. However, a significant correlation ( $r=0.000-0.038$ ) was reported between the original distance of PFA and those of medial circumflex femoral artery (MCFA) and lateral circumflex femoral artery (LCFA) in the right and left male limbs, as well as in right female limbs, while no significant correlation was recorded in that of the left female limbs.

The patterns of origin of the MCFA were listed (Fig. 5). In pattern I, the MCFA originated from the medial side of 
Table 2. Distance of the origin of profunda femoris and its circumflex femoral branches $(n=90)$

\begin{tabular}{lccccccc}
\hline Original distance & \multicolumn{3}{c}{ Male limbs } & & \multicolumn{3}{c}{ Female limbs } \\
\cline { 2 - 3 } & Right (25) & Left (25) & Both (50) & & Right (20) & Left (20) & Both (40) \\
\hline PFA0-MI & $51.5 \pm 1.9$ & $49.7 \pm 1.9$ & $50.6 \pm 1.3$ & & $48.5 \pm 2.2$ & $48.9 \pm 2.2$ & $48.8 \pm 1.5$ \\
PFAL & $16.1 \pm 1.96$ & $16.5 \pm 2.1$ & $16.13 \pm 1.4$ & & $20.5 \pm 2.8$ & $23 \pm 2.4$ & $21.7 \pm 1.8$ \\
PFAO-MCFAO & $18.6 \pm 2.1$ & $19.6 \pm 1.9$ & $19.1 \pm 1.4$ & & $18.8 \pm 2.7$ & $19.1 \pm 2.1$ & $18.9 \pm 1.7$ \\
PFAO-LCFAO & $20.2 \pm 2.2$ & $22.5 \pm 2.3$ & $21.3 \pm 1.6$ & & $21 \pm 1.6$ & $21.7 \pm 2.6$ & $21.3 \pm 1.8$ \\
\hline
\end{tabular}

Data are expressed as mean \pm SEM $(n=90)$. No sex or side significant difference was observed at all measurements; PFAO-MI — distance between origin of profunda femoris artery and midpoint of inguinal ligament; PFAL — profunda femoris artery length; PFAO-MCFAO — distance between profunda femoris origin and medial circumflex femoral origin; PFAO-LCFAO - distance between origin of profunda femoris and lateral circumflex femoris origin
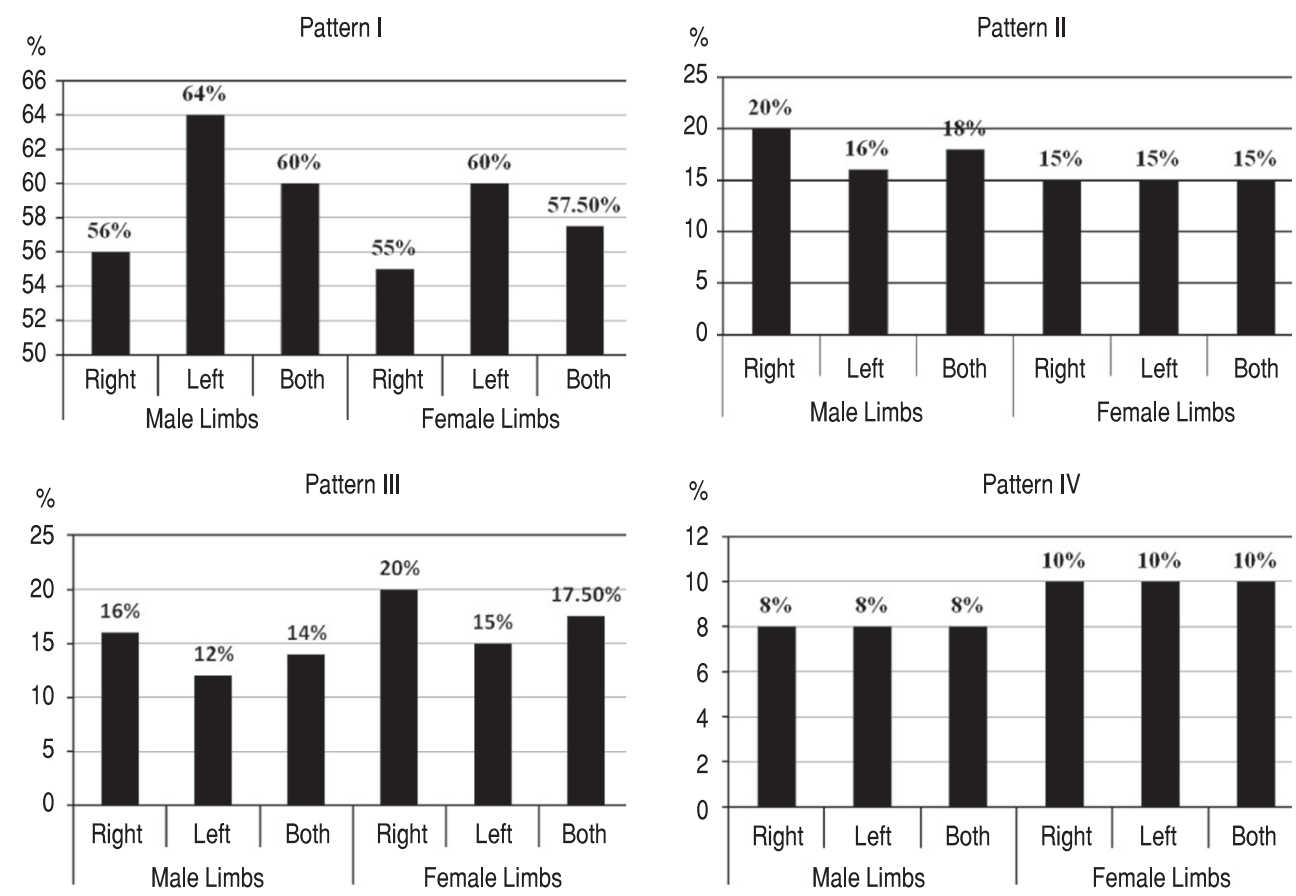

Figure 5. Original patterns of medial circumflex femoral artery (MCFA). Pattern I: from profunda femoris artery (PFA); Pattern II: from femoral artery (FA) by a common trunk with PFA; Pattern III: from FA superior to the origin of PFA; Pattern IV: from superficial femoral artery.

the PFA (Fig. 2). The incidence of this pattern was reported in $59 \%$ of all limbs, $60 \%$ of male $(56 \%$ on right, $64 \%$ on left) and $57.5 \%$ of female (55\% on right, $60 \%$ on left) limbs. In pattern II, the MCFA originated from the FA by a common stem with the PFA (Fig. 6). This pattern was noticed in $18 \%$ of the male limbs ( $20 \%$ on right, $16 \%$ on left) and $15 \%$ of the female limbs. In pattern III, the MCFA arose from the FA superior to the origin of the PFA (Fig. 1). The incidence of this pattern was observed in $14 \%$ of male (16\% on right, $12 \%$ on left) and $17.5 \%$ of female limbs ( $20 \%$ on right, $15 \%$ on left limbs). In pattern IV, the MCFA originated from the superficial femoral artery (SFA) (Fig. 3). This pattern was seen in $8 \%$ of male and $10 \%$ of female limbs. Statistically, no significant differences were reported between the patterns of origin of MCFA of right and left male limbs, right and left female limbs, right male and right female limbs, left male and left female limbs, total male and total female limbs ( $p>0.05)$.

Four original patterns of the LCFA were listed (Fig. 7). The LCFA originated from the lateral aspect of the PFA (pattern I) in $74 \%$ of male and $65 \%$ of female limbs (Figs. 2, 3). The incidence of pattern II, where the LCFA originated as a common stem with PFA from FA (Figs. 1, 6) was observed in $14.4 \%$ of all limbs, $14 \%$ of male and $15 \%$ of female limbs, while the incidence of pattern III, where the LCFA originated from the FA above the level of the origin of the PFA (Fig. 8), was noticed in $10 \%$ of all limbs, $8 \%$ of male and $12.5 \%$ of 


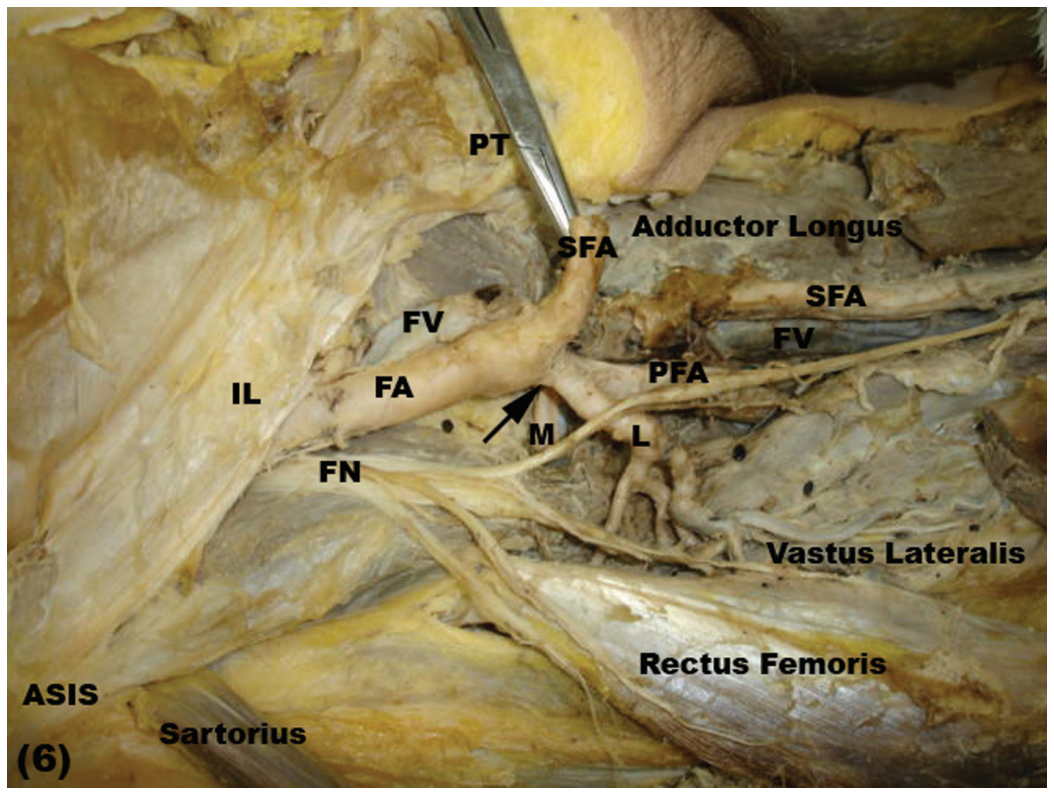

Figure 6. A right female lower limb showing both medial $(\mathrm{M})$ and lateral $(\mathrm{L})$ circumflex femoral branches originating from the femoral artery (FA) as a common stem (arrow) with the profunda femoris artery (PFA); FV — femoral vein; FN — femoral nerve; IL — inguinal ligament; SFA — superficial femoral artery; PT — pubic tubercle; ASIS — anterior superior iliac spine.
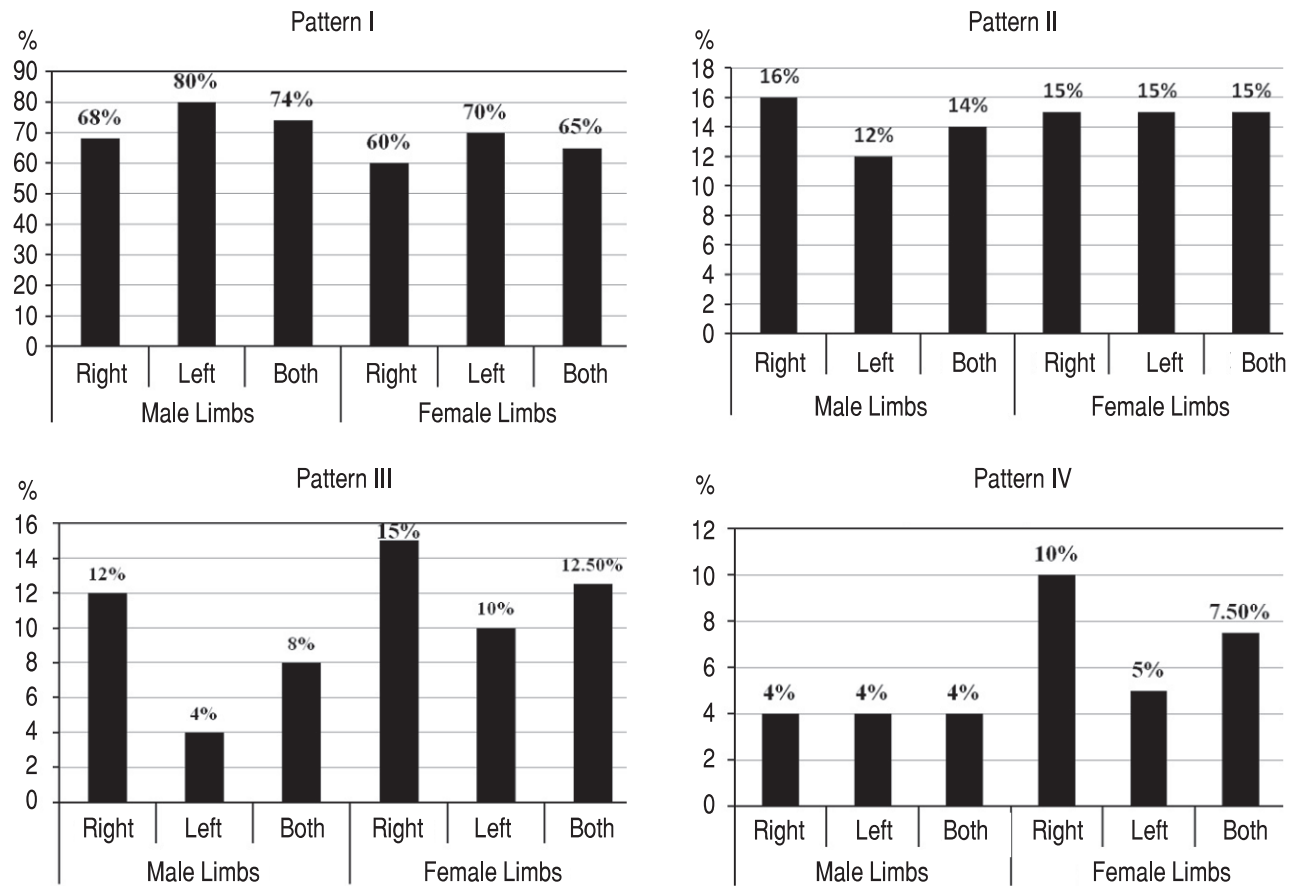

Figure 7. Original patterns of lateral circumflex femoral artery (LCFA). Pattern I: from lateral aspect of profunda femoris artery (PFA); Pattern II: from femoral artery (FA) by a common stem with PFA; Pattern III: from FA above the original level of PFA; Pattern IV: from superficial femoral artery.

female limbs. In pattern IV, the LCFA originated from the SFA (Fig. 4) in $4 \%$ of male and $7.5 \%$ of female limbs. Statistically, no significant differences were reported between the original patterns of LCFA in the right nor the left male limbs ( $p>0.05)$.
The range of the original distance of PFA and its medial and lateral circumflex branches was listed (Table 3). The range of the distance between the origin of PFA and the midpoint of inguinal ligament was $25-67 \mathrm{~mm}$ in male and $28-70 \mathrm{~mm}$ in female limbs. Moreover, the 


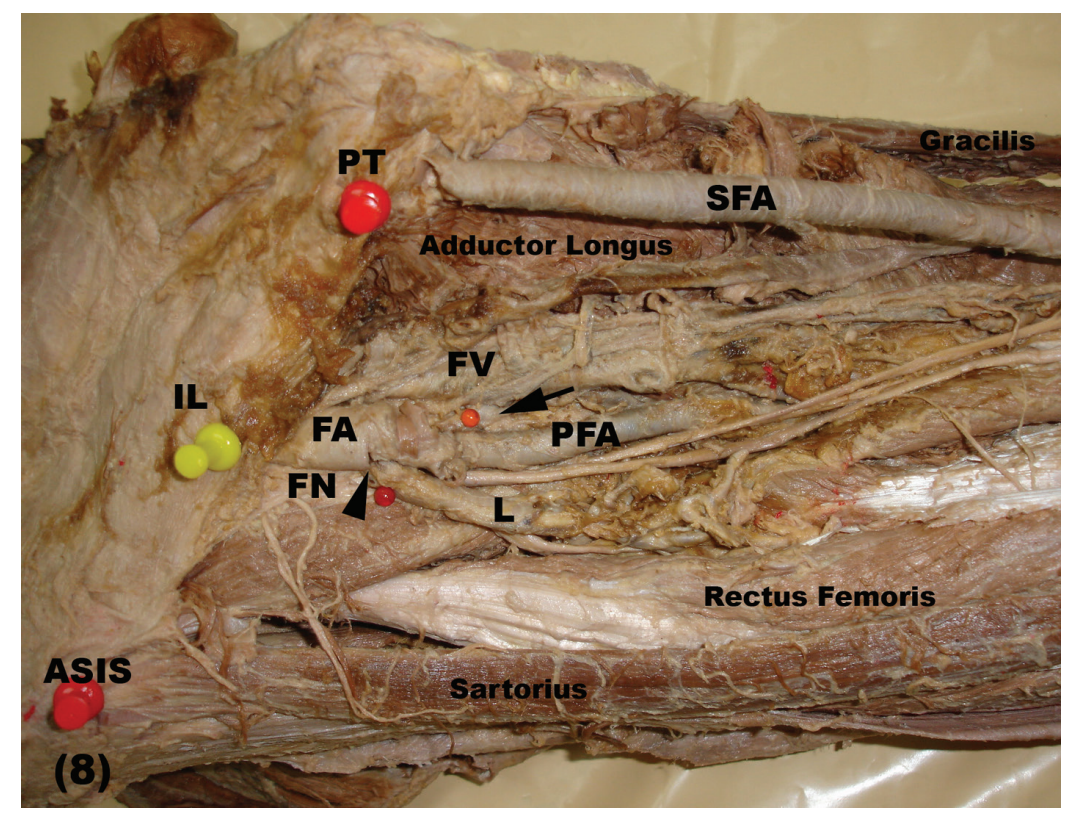

Figure 8. A right female lower limb showing the lateral circumflex femoral artery (L) originating from the lateral side (arrow head) of femoral artery (FA), while the medial circumflex femoral branch (arrow) originates from the medial aspect of the profunda femoris artery (PFA); SFA — superficial femoral artery; IL — inguinal ligament; PT — pubic tubercle; ASIS — anterior superior iliac spine; FV — femoral vein; FN — femoral nerve.

Table 3. Distance of origin of PFA, MCFA and LCFA in male and female limbs

\begin{tabular}{|c|c|c|c|c|c|c|c|c|c|c|c|c|}
\hline \multirow{3}{*}{$\begin{array}{l}\text { Distance } \\
\text { of origin }\end{array}$} & \multicolumn{4}{|c|}{ PFA } & \multicolumn{4}{|c|}{ MCFA } & \multicolumn{4}{|c|}{ LCFA } \\
\hline & \multicolumn{2}{|c|}{ Male (50) } & \multicolumn{2}{|c|}{ Female (40) } & \multicolumn{2}{|c|}{ Male (50) } & \multicolumn{2}{|c|}{ Female (40) } & \multicolumn{2}{|c|}{ Male (50) } & \multicolumn{2}{|c|}{ Female (40) } \\
\hline & Right & Left & Right & Left & Right & Left & Right & Left & Right & Left & Right & Left \\
\hline $0-10 \mathrm{~mm}$ & 0 & 0 & 0 & 0 & 7 & 5 & 3 & 3 & 4 & 3 & 2 & 3 \\
\hline $11-20 \mathrm{~mm}$ & 0 & 0 & 0 & 0 & 6 & 7 & 5 & 6 & 8 & 9 & 6 & 7 \\
\hline $21-30 \mathrm{~mm}$ & 1 & 0 & 0 & 1 & 4 & 5 & 5 & 4 & 5 & 6 & 5 & 4 \\
\hline $31-40 \mathrm{~mm}$ & 12 & 10 & 9 & 8 & 2 & 3 & 3 & 3 & 4 & 5 & 4 & 3 \\
\hline $41-50 \mathrm{~mm}$ & 6 & 8 & 6 & 7 & 0 & 0 & 0 & 0 & 0 & 0 & 0 & 0 \\
\hline $51-60 \mathrm{~mm}$ & 5 & 7 & 5 & 4 & 0 & 0 & 0 & 0 & 0 & 0 & 0 & 0 \\
\hline $61-70 \mathrm{~mm}$ & 0 & 0 & 0 & 0 & 0 & 0 & 0 & 0 & 0 & 0 & 0 & 0 \\
\hline From FA & 0 & 0 & 0 & 0 & 4 & 3 & 3 & 2 & 3 & 1 & 2 & 1 \\
\hline From SFA & 0 & 0 & 0 & 0 & 2 & 2 & 1 & 2 & 1 & 1 & 1 & 2 \\
\hline Total & 25 & 25 & 20 & 20 & 25 & 25 & 20 & 20 & 25 & 25 & 20 & 20 \\
\hline
\end{tabular}

PFA - distance of origin of profunda femoris artery from midpoint of inguinal ligament; MCFA — distance of origin of medial circumflex femoral artery from origin of PFA; LCFA - distance of origin of lateral circumflex femoral artery from origin of PFA; FA — femoral artery (common femoral artery); SFA — superficial femoral artery

MCFA arose from the PFA at a distance of $0-42 \mathrm{~mm}$ in male limbs and 0-40 $\mathrm{mm}$ in female limbs. However, the range of the distance of the LCFA origin was $0-45 \mathrm{~mm}$ in both male and female limbs.

\section{DISCUSSION}

Knowledge of the site and pattern of origin of the PFA and its circumflex branches is of great clinical importance in performing surgical or radiologi- cal intervention of the femoral region to avoid the iatrogenic complications. In the present study, the PFA originated mostly from the posterolateral side of the FA in $42.2 \%$ of the limbs with no sex or side difference. These findings agree with the studies of Dixit et al. [8] and Prakash et al. [15] who stated that the incidence of the posterolateral origin of the PFA from the FA was seen in $42.1 \%$ and $50 \%$ of the limbs, respectively. However, a lower incidence rate, 13.3\%, 
$18.3 \%, 30.4 \%$ and $30.8 \%$, were previously reported in the literature for this original pattern of PFA [11, 16, $17,20]$. Moreover, in $25.6 \%$ of the cases in present study, the PFA originated from the posterior aspect of the FA with no side difference, but with a relative higher incidence in the female limbs. In agreement with the results of present study, Dixit et al. [8] found this origin pattern in $28.5 \%$ of the limbs. However, a higher incidence rates were recorded in the literature, 53.3\% [11], 46.9\% [15], 46.1\% [17] and $44.6 \%$ [20]. Moreover, the PFA originated from the lateral aspect of FA in $20 \%$ of the limbs with no side or sex differences in the present study and this in agrees with Siriporn et al. [20] who found this pattern in $21.4 \%$ in Thailand population. In $12.2 \%$ of the limbs, the PFA originated from the posteromedial side of FA with male and left side predominance in the present study. Similarly, the origin of PFA from the medial side of FA was found in $10.5 \%$ [8], while it was reported in Thailand (3.6\%) [20] and Indians (3\%) [15]. The variations in the site of origin of PFA might be due to the variability in the pattern of regression of the rete femorale [14].

The distance between the origin of PFA and the midpoint of the inguinal ligament was 25-67 $\mathrm{mm}$ in male limbs and $28-70 \mathrm{~mm}$ in female limbs and its mean measurement was $51.5 \pm 1.9 \mathrm{~mm}$ on the right side and $49.7 \pm 1.9 \mathrm{~mm}$ on the left side in male limbs and $48.5 \pm 2.2 \mathrm{~mm}$ on the right side and $48.9 \pm 2.2 \mathrm{~mm}$ on the left side in female limbs. In disagreement with the results of present study, different ranges of the distance were obtained in literature: $31-40 \mathrm{~mm}$ in right limbs and 41-50 $\mathrm{mm}$ in left limbs [8]; $55 \mathrm{~mm}$ on right side and $45 \mathrm{~mm}$ on left side [17]; commonly between 31-40 mm [2] and its mean was $42 \mathrm{~mm}$ [15], $44 \mathrm{~mm}$ [18], $32 \mathrm{~mm}$ [16] and its normal range was 35-40 mm [21]. Awareness of the original site and distance of PFA is important for prevention of iatrogenic injuries that may occur during the diagnostic or therapeutic procedures in the femoral region.

MCFA is an important branch in supplying blood to the head and neck of femur, fat of acetabulum and adductor muscles [21]. It has a great clinical significance in flap plastic surgery as well as in selective arteriography in cases of idiopathic ischaemic necrosis of the femoral head [13]. MCFA mostly originated from PFA and to lesser extent from the FA [22]. Comparing the data of the present study with the previous literature, different results were obtained. In the present study, MCFA originated mostly from the medial side of the PFA in $58.9 \%$ of the limbs with left side and male predominance. This result was $59.5 \%$ found by Lalovic et al. [12], $56 \%$ on right and $66.7 \%$ on left side reported by Dixit et al. [8], 62\% stated by Samarawickrama et al. [17], 63\% found by Siddharth et al. [18], 63.2\% found by Adachi [1], and 67.2\% reported by Prakash et al. [15]. However, higher percentages were reported by Tanyeli et al. [23] in 79\%, Massoud and Fletcher [14] in 81\%, Vasquez et al. [25] in 78.8\%, and Anwer et al. [2] in 73.33\%. Meanwhile, Basar et al. [4] in their angiographic study found the normal original pattern of MCFA in $40.9 \%$ of the cases.

In the present study the pattern of origin of MCFA from FA was subdivided into 2 main patterns: the first pattern determined the origin of MCFA from the common FA superior to the origin of PFA and the second one reported those originated from SFA inferior to the origin of PFA. Thus, MCFA originating from FA superior to PFA origin was found in $15.6 \%$ of the cases (14\% of male, $17.5 \%$ of female limbs) and that originating from FA inferior to the origin of PFA was observed in $8.9 \%$ of the limbs ( $8 \%$ of male, $10 \%$ of female limbs). In agreement with the results of the present study, Dixit et al. [8] found that MCFA originated from FA in $15.3 \%$ superior to the origin of PFA and in $6.6 \%$ inferior to the origin of PFA with right side predominance. Adachi [1] found the later pattern in 14\%, Siddharth et al. [18] in 26\%, Massoud and Fletcher [14] in 6.4\%, Basar et al. [4] in 48.9\%, Tanyeli et al. [23] in 15\%, Prakash et al. [15] in 32.8\%, Samarawickrama et al. [17] in 31\% and Anwer [2] in $21.67 \%$ of the cases.

In the present study, origin of MCFA from FA in a common stem with PFA was seen in $8.9 \%$ of the limbs ( $8 \%$ of male, $10 \%$ of female limbs). However, this origin pattern was rarely described in the literature, where it was found in $2.4 \%$ [12] and in $4 \%$ of cases [8, 17].

The anatomic relationships of MCFA agreed with the data published in the literature $[12,21,25]$ with exception of one case that showed MCFA originating from the medial aspect of SFA and passed superficial to the femoral vein. Similarly, Chitra [5] found 1 case of MCFA originating from FA and coursed in front of the femoral vein.

In the present study the distance between the branching point of MCFA and the original point of PFA was measured. The mean measurement of this distance was $19.1 \pm 1.4 \mathrm{~mm}$ in male and $18.9 \pm 1.7 \mathrm{~mm}$ in female limbs and these results agreed with the values mentioned in the literature as $20 \mathrm{~mm}$ [15] and 
$24.4 \mathrm{~mm}$ [2] in Indian population. Moreover, the present study revealed a significant side but with no sex difference, where the mean of this distance measured $18.6 \pm 2.1 \mathrm{~mm}$ in the right male, $19.6 \pm 1.9 \mathrm{~mm}$ in the left male, $18.8 \pm 2.7 \mathrm{~mm}$ in the right female and $19.1 \pm 2.1 \mathrm{~mm}$ in the left female limbs. Meanwhile, Tanyeli et al. [23] reported that, the mean distance between the origin of MCFA from FA and midpoint of inguinal ligament was $25 \pm 14 \mathrm{~mm}$. In the present study, the original distance of MCFA from the origin of PFA was mostly between 0-10 mm in male and $11-20 \mathrm{~mm}$ in female limbs with a range of $0-40 \mathrm{~mm}$ in both male and female limbs. However, the MCFA originated mostly from the PFA at a range of distance $0-10 \mathrm{~mm}$ [14], 11-30 [3, 6, 23] on both sides and sexes.

The LCFA originated mostly from the lateral side of PFA and in some cases from FA, including the common stem with PFA [19]. Adachi [1] found that LCFA originated from PFA or FA in $78.2 \%$ and $13.8 \%$ of cases, Siddharth et al. [18] in 71\% and $16 \%$, Massoud and Fletcher [14] in $81 \%$ and $2.8 \%$ in their angiographic study, Basar et al. [4] in $67.1 \%$ and $32.9 \%$, Fukuda et al. [9] in $78.2 \%$ and $21.4 \%$ in their angiographic study, Choi et al. [6] in $86.8 \%$ and $13.2 \%$, Vasquez et al. [25] in $78.8 \%$ and $21.2 \%$ of cases, Tansatit et al. [22] in $56.67 \%$ and $43.33 \%$ of cases, Uzel et al. [24] in $77.3 \%$ and $22.7 \%$ of cases, Samarawickrama et al. [17] in $92.3 \%$ from PFA and $8 \%$ from common trunk with no from FA, Prakash et al. [15] in $81.25 \%$ and $18.75 \%$ of cases, Dixit et al. [8] in $72.8 \%$ in right limbs, $77.2 \%$ in left limbs and $27.2 \%$ in right, $22.8 \%$ in left limbs, Anwer et al. [2] in $83.33 \%$ and $15 \%$ of cases, and Sabnis [16] in $80 \%$ and $16.6 \%$ of cases including the common trunk with PFA. These differences might be in part related to the number of the limbs used in each study or due to an ethnic factor.

In the present study, the LCFA arose from lateral aspect of PFA in $74 \%$ of male and $65 \%$ of female limbs with left side predominance in both male $(80 \%)$ and female $(70 \%)$ limbs and from FA in $26 \%$ of male and $35 \%$ of female limbs, including the common stem with PFA. In $15.6 \%$ of the specimens LCFA originated from $F A$; in $10 \%$ of them LCFA originated from the common FA above the origin of PFA with right side and female predominance, while in the other $5.6 \%$ of cases it arose from the SFA below the origin of PFA with female predominance. These results were in accordance with the data published by different authors $[4,8,9,18,24]$.
Moreover, in the remaining $14.4 \%$ of the specimens, the LCFA originated from FA in a common trunk with PFA or/and MCFA with right side predominance. These findings agreed with that of Dixit et al. [8]. In disagreement with the present study, the LCFA originated from the FA as a common trunk with MCFA in $14.3 \%$, with PFA in $10.7 \%$ and as a trifurcation with PFA and MCFA in $7.1 \%$ in black Kenyan population [19], from a common trunk with PFA in $1.7 \%$ in Indian population [2], from a common trunk with PFA and MCFA in $0.9 \%$ in Turkish population [24] and from a common trunk with PFA and MCFA in 5\% [18]. These differences may be related to the number of specimens or the species of the cadavers.

The mean of the distance of the origin of LCFA from PFA origin in the present study was found to be $20.2 \pm 2.2 \mathrm{~mm}$ in right male and $22.5 \pm 2.3 \mathrm{~mm}$ in left male limbs. In the female cadavers, it was $21 \pm 1.6 \mathrm{~mm}$ in right and $21.27 \pm 2.6 \mathrm{~mm}$ in left-side limbs. Similar results $(21.2 \mathrm{~mm}$ and $25 \mathrm{~mm}$ ) were reported by Anwer et al. [2] and Prakash et al. [15]. However, different results were found in some literature, where a higher value $(48 \pm 12 \mathrm{~mm})$ was observed in Turkish population [24] and lower values (15 $\mathrm{mm}$ and $21-30 \mathrm{~mm}$ ) were reported in Indian population $[3,8,18]$.

In present study, LCFA originated from PFA at a distance of 11-20 mm in most of the cases. However, the average of this distance was commonly between 21 and $30 \mathrm{~mm}$ on both sides $[2,3,8,17]$. The distance between the origin of LCFA and original point of PFA has an important clinical significance especially in surgical or angiographic interventions and so these distances may be useful for health professionals dealing with PFA and its circumflex femoral branches. In our study, the incidence of MCFA originating from FA was higher than that of LCFA. This difference might be associated with the distal shift of the level of separation of PFA from FA $[10,15]$.

The lower limb arteries develop from the axial artery which is derived from the fifth lumber artery. This axial artery runs on the dorsal aspect of the pelvis and thigh lateral to the sciatic nerve. At later developmental stage, this axial artery differentiates into 3 main parts: proximal part that gives the sciatic artery, middle part down to the knee that gives the deep popliteal artery and distal part that gives the interosseous artery. Proximal to the axial artery, the external iliac artery sprouts from the umbilical 
artery. Both inferior epigastric and proximal part of femoral arteries develop from the external iliac artery. The middle part of the femoral artery and the profunda femoris branch develop from the ventral arterial plexus called rete femorale, while the distal part of the femoral artery develops from ramus communicans superior of the sciatic artery. Simultaneously with the development of these arteries, the sciatic artery disappears for the great part. Due to this complex developmental process, many congenital anomalies and variations of the arteries in pelvis and lower limb have been documented. Congenital variations of the PFA occur due to the numerous possible patterns of regression of rete femorale $[7,10]$.

\section{CONCLUSIONS}

Knowledge of the normal and variant sites and distance of the origin of PFA and its circumflex branches are not only of great clinical importance during diagnostic and operative interventional procedures but also helps in reduction of the intra-operative and post-operative complications in femoral region.

\section{ACKNOWLEDGEMENTS}

Great appreciations to all technical members of the Department of Anatomy, Faculty of Medicine, King Abdulaziz University for their help during doing this work.

\section{REFERENCES}

1. Adachi B (1928) Das arterien system der Japaner, Band II. Verlag der Kaiserlich, Kyoto, pp. 151.

2. Anwer D, Arun SK, Humbarwadi RS (2013) A study of variation in origin of profunda femoris artery and its branches. Int J Biomed Adv Res, 4: 366-368.

3. Baptist M, Sultana F, Hussain T (2007) Anatomical variations: the origin of profunda femoris, its branches and diameter of the femoral artery. Professional Med J, 14: 523-527.

4. Basar R, Sargon MF, Cumhur M, Bayraoglu A, Demiryurek D (2002) Distinct intergender difference in the femoral artery ramification patterns found in the Turkish population: angiographic study. Anat Sci Int, 77: 250-253.

5. Chitra R (2008) A rare variational anatomy of the profunda femoris artery. Folia Morphol, 67: 157-158.

6. Choi SW, Park JY, Hur MS, Park HD, Kang HD, Hu KS, Kim HJ (2007) An Anatomic assessment of perforators of the lateral circumflex femoral artery for anterolateral thigh flap. J Craniofac Surg, 18: 866-871.

7. Datta AK (2005) Essentials of human embryology. In cardiovascular system. $5^{\text {th }}$ Ed. Current Books International, pp. 196-197.

8. Dixit D, Kubavat DM, Sureshbhai PR, Mital MP, Tulsibhai CS (2011) A study of variations in the origin of profunda femoris artery and its circumflex branches. Int J Biol Med Res, 2: 1084-1089.

9. Fukuda $H$, Ashida M, Ishil R, Abe S, Ibukuro K (2005) Anatomical variants of the lateral femoral circumflex artery: an angiographic study. Surg Radiol Anat, 27: 260-264.

10. Keen JA (1961) A study of the arterial variations in the limbs with special reference to symmetry of vascular patterns. Am J Anat, 108: 245-261.

11. Kulkarni S, Nikade V (2013) A study of branching pattern of femoral artery in femoral triangle in cadavers. Int J Recent Trends Technol, 6: 53-55.

12. Lalovic N, Millos M, Milan K, Radovan C, Milan S, Marko I (2013) origin of the medial circumflex femoral artery: a cadaveric study. Med Glas (Zenica), 10: 198-202.

13. Locke MB, Zhong T, Mureau MA, Hofer SO, Tug ' $O$ ' war (2012) challenges of transverse upper gracilis (TUG) myocutaneous free flap breast reconstruction. J Plast Reconstr Aesthet Surg, 65: 1041-1050.

14. Massoud TF, Fletcher EW (1997) Anatomical variants of the profunda femoris artery: an angiographic study. Surg Radiol Anat, 19: 99-103.

15. Prakash KJ, Kumar BA, Betty AJ, Kumar YS, Singh G (2010) Variations in the origins of profunda femoris, medial and lateral femoral circumflex arteries: a cadaveric study in the Indian population. Romanian J Morphol Embryol, 51: 167-170.

16. Sabnis AS (2013) Anatomical variations of profunda femoris artery. J Clin Res Letters, 4: 54-56.

17. Samarawickrama MB, Nanayakkara BG, Wimalagunarathna KWR, Nishantha DG, Walawage UB (2009) Branching pattern of the femoral artery at the femoral triangle: a cadaver study. Galle Med J, 14: 31-34.

18. Siddharth P, Smith NL, Mason RA, Giron F (1985) Variational anatomy of the deep femoral artery. Anat Rec, 212: 206-209.

19. Sinkeet SR, Ogeng'o JA, Elbusaidy H, Olabu BO, Irungu MW (2012) Variant origin of the lateral circumflex femoral artery in a black Kenyen population. Folia Morphol, 71: 15-28.

20. Siriporn T, Thanaporn R, Chollada V (2012) The origin of profunda femoris artery in Thais. Siriraj Med J, 64 (suppl. 1): S34-S36.

21. Standring $S$ (2008) Pelvic girdle, gluteal region and hip joint, profunda femoris artery. In: Gray's anatomy. The anatomical basis of clinical practice. $40^{\text {th }} \mathrm{Ed}$. Elsevier Churchill Livingstone, pp. 1379-1380.

22. Tansatit T, Wanidchaploi S, Sanguansit P (2008) The anatomy of the lateral circumflex femoral artery in the anterolateral thigh flap. J Med Assoc Thai, 91: 1404-1409.

23. Tanyeli E, Uzel M, Yildirim M, Celik HH (2006) An anatomical study of the origins of the medial circumflex femoral artery in Turkish population. Folia Morphol, 65: 209-212.

24. Uzel M, Tanyeli E, Yildirim M (2008) An anatomical study of the origins of the lateral circumflex femoral artery in the Turkish population. Folia Morphol, 67: 226-230.

25. Vazquez JM, Murillo J, Maranillo E, Parki, Sanudo J (2007) Patterns of the circumflex femoral arteries revised. Clin Anat, 20: 180-185. 